\title{
A Performance-Based Pavement Management System for the Road Network of Montreal City - a Conceptual Framework
}

\author{
Md. Shohel Reza Amin ${ }^{1}$, Luis E. Amador-Jiménez ${ }^{2}$ \\ ( ${ }^{1}$ Research Assistant, Dept. of Building, Civil and Environmental Engineering, Concordia \\ University, email:md_amin@encs.concordia.ca) \\ $\left({ }^{2}\right.$ Assistant Professor, Dept. of Building, $\bar{C}$ ivil and Environmental Engineering, Concordia \\ University. email: amador@encs.concordia.ca)
}

\begin{abstract}
Arterial roads of Montreal city, mostly constructed in 1950's, are at an advanced state of deterioration and need major rehabilitation, upgrading, or even reconstruction. City of Montreal has allocated over \$1.6 billion for road infrastructure in its 2012-2014 Three-year Capital Work Program. This investment can be wasted without proper infrastructure asset management system. The current practice of mill and asphalt overlay method by City of Montreal to rehabilitate the pavement is inadequate to repair potholes, fatigue and cracking. A performance-based pavement management system can predict the response and performance of pavement under actual dynamic traffic loads. As of today, implementations of pavement management systems (PMS) are dedicated to achieve optimal levels of condition under budget restrictions. Other important objectives (e.g. mobility, safety, accessibility and social cost), along with investments to upgrade and expand the network, are normally left outside the modeling. This paper presents a conceptual framework of a dynamic PMS for the road network of Montreal City. This dynamic PMS will manage continuous aggregate behavior of transportation system and can solve optimization problems of pavement management at any time interval.
\end{abstract}

Keywords: Pavement Management System, Dynamic, Performance Modeling, Optimization.

\section{INTRODUCTION}

The pavement management systems is an approach that incorporates the economic assessment of trade-offs between competing alternatives [1, 2]. Historically, pavement management tools such as PAVER and HDM3 [3] were based on cost-benefit analysis incapable of trading-off decisions across asset types and modes of transportation [4]. The adaptation of linear programming and other heuristic optimization techniques for asset management came to address most of these issues $[5,6]$. These techniques are capable of finding the optimal path to take full advantage of cost-effectiveness of individual treatments, associated with individual asset elements, and the benefits of advancing or deferring a certain treatment [1,7]. However, the economic impact of multiple strategies (i.e., rehabilitation versus capital investments) has not yet been addressed for safety, pavement condition, congestion, pollution and social cost. The perception of congestion combined with pavement condition, highway capacity, accessibility, financial incentives, personal safety, and to a lesser degree environmental responsibility impacts personal choices of modes, routes and links [8]. Periodic incorporation of choice models into the pavement management system (PMS) will not only render it more tractable mathematically and more consistent with most growth-theory frameworks and distribution models, but also provide a better way of depicting on-going aggregate behavior and a more satisfactory PMS [8]. 
The objective of this paper is to outline a conceptual framework of a pavement management system for the road network of Montreal City incorporating dynamic states of land use, traffic volumes, design capacities, and pavement conditions of arterial roads. Most of the arterial roads of Montreal city are constructed in 1950's and are at an advanced state of deterioration. The commonly cited factors of this advanced state of deterioration are improper maintenance, low priority on infrastructure maintenance, inadequate funding, and the use of poor materials in the original construction. The City council seems to focus more on improving patching technology and execution, which don't appear to survive beyond two to five years. Moreover, the investments of City of Montreal on road system have increased more than $560 \%$ since 2001. The Three-year Capital Works Program (TCWP) 2012-2014 allocated over \$130 million for maintaining and upgrading the city's roads, including $\$ 2.5$ million that will be used solely for minor bridge and tunnel repairs [9]. A performance-based PMS can predict the response and performance of pavement under actual dynamic traffic loads, and can ensure cost effective scheduling of maintenance and rehabilitation operations.

\section{MEASUREMENT OF THE ROAD INFRASTRUCTURE PRODUCTIVITY}

The pavement investment policies face important policy questions regarding the uses of pavement maintenance and rehabilitation (M\&R) funds such as: What is the optimal level of pavement M\&R funding? How can the need for this level of $M \& R$ funding be validated persuasively? What choices of $M \& R$ sections in the current budget period would most effectively move pavement conditions toward an optimal level in the long-term? [10] There is a wide range of options to achieve an appropriate investment policy. They are: construction with a low initial cost followed by frequent low cost strengthening by overlays, construction of high quality pavements with higher initial costs but lower future maintenances costs, and construction of higher strength initial pavement followed by frequent thin overlays [11,12]. The first option is applicable when initial capital is limited but a steady flow of maintenance funds are available, however, the second and third options are supported by optimization and design studies.

The productivity of a road infrastructure needs to be understood before developing the optimization and design studies for pavement investment strategies. There is no universally accepted methodology to measure the productivity of a road infrastructure probably because of the lack of methodological consensus and the complexity of the hierarchical characteristics of many sub-systems within the road infrastructure system [13]. The development of cost efficiency analysis methods and methods for analyzing deterioration, maintenance and renewal under a budget constraint is necessary [13]. An economic optimization model, with given budget constraints, can differentiate different maintenance scenarios while identifying optimal actions and scheduling schemes [13].

The economic optimization model for PMS consists of pavement deterioration model; user cost model; and construction, maintenance and rehabilitation cost model under possible budgets constraints [10]. Life-cycle cost analysis (LCCA), which is associated with the evaluation of an asset with a definite life expectancy, is an appropriate economic optimization model to examine the subsequent maintenance works on the pavement [11]. 


\section{STUDIES ON LIFE-CYCLE COST ANALYSIS (LCCA)}

LCCA is a tool to assess total cost of M\&R operations, to distinguish between alternatives, and to provide a basis for identifying trade-offs related to alternatives [14]. LCCA for PMS have been applied in a number of studies [3, 15]. Uddin et al. [15] describe a LCCA program developed for the Pennsylvania Department of Transportation (DOT). The program economically evaluates a range of strategies for design and rehabilitation of road pavements by applying BASIC and FORTRAN components. However, the pavement deterioration algorithm is somehow simplistic [15].

Haas and Hudson [2] suggest a pavement management working system, including pavement deterioration prediction, decision optimization and feedback, and minimum serviceability index. The minimum serviceability index can be computed by Markov decision process (MDP) which may also minimize the long-term maintenance costs [16]. Kher and Cook [17] describe the Program Analysis of Rehabilitation System (PARS) model developed by the Ontario Ministry of Transportation and Communication. The application of maintenance treatments and action timings can also be optimized by MDP [16].

The Texas Transportation Institute has developed optimization models to allocating M\&R funds and other resources among highway districts. Under the budget constraints, the models attempt to maintain the quality of the road segments to some pre-specified level [16]. The use of a MDP would take a different approach to optimization since the specified quality of road segments would be calculated in determining the optimal (minimum cost) maintenance policy; an optimum (minimum) budget would then be computed [16]. Scullion and Stein [18] use the pavement deterioration matrices in planning $M \& R$ needs, however, the threshold levels of pavement deterioration for taking certain maintenance actions are determined exogenously [16].

Artman and Liebman [19] develop a dynamic optimization programming to schedule the rehabilitation projects of the airfield pavement. The rehabilitation projects are scheduled by maximizing the area under a utility-weighted pavement condition (PCR) versus time curve. The optimization programming is somehow similar to MDP; however, it uses the maximization of utility function of pavement condition rather than minimization of cost measures. Another drawback of this method is that the pavement condition rating (PCR) prediction is based on the regression analysis, which does not include current or recent pavement condition data [16].

The discussion of the above-mentioned studies explains that the optimization of PMS can better be addressed by MDP, as it results in optimal solution of preventive maintenance strategies depending on the pavement conditions. The conventional MDP applies an infinite horizon probabilistic dynamic programming (DP) to solve the optimization problems in which LCCA is performed on the basis of long-term behavior of the pavement structure [20].

The applications of MDP for optimization problems of PMS are justifies by various studies. Golabi et al. [21] determine the implications of implementing MDP for the Arizona DOT. Golabi et al. [21] identify that a total of $\$ 40$ million was saved from the road preservation budget because of applying MDP optimization method. The Arizona MDP model applies twosteps linear programming to get a steady-state solution. A set of steady state maintenance actions was calculated to minimize the expected long-term average costs. If a road was found to be in a particular state, there was a specified probability that a particular maintenance action would be taken. Golabi et al. [21] argue that the PMS has provided a 'defensible procedure for preparing one-year and five-year budgets' and has helped to 'justify the revenue requests before oversight legislative committees.' However, the maintenance policy was found to be stationary, and random and long-term performance standards were not included [16]. 
Carnahan et al. [22] develop MDP model for optimal decision-making capability of PAVER, which is a PMS developed by the U.S. Army Corps of Engineers Construction Engineering Research Laboratory (CERL). Unlike Arizona MDP model, this model incorporates pavement data as a pavement condition rating (PCR) based inventory at a single point in time. A transition matrix is developed to identify a Markov chain for each pavement type [16]. Feighan et al. [23] also employed dynamic programming in conjunction with a Markov chain probabilitybased prediction model to obtain minimum cost maintenance strategies over a given LCCA period.

\section{PAVEMENT PERFORMANCE MODELING}

The appropriate and effective pavement performance models are the foundation for the long-term analysis of PMS. The performance models calculate the future condition of the pavement based on which PMS optimizes several maintenance treatments in the long-term maintenance process. The performance models have significant features in the PMS such as (1) the performance prediction models are used when the prioritization of maintenance treatments is required for each segments of road network [24], (2) the models enable the transportation agencies to estimate long-range investment requirement during the life-span of the pavement [24], (3) the models also estimate the consequences of budget allocation for maintenance treatments of a particular road segment on the future pavement condition of that road segment [24], (4) the models can be applied for life-cycle economic evaluation of the pavement as they relate the pavement exposure variables to pavement deteriorations in the performance indicators [24], (5) many components of PMS such as pavement structural design, maintenance treatment strategies, and priority programming are directly related to the output of the pavement performance prediction (PPP) models [25].

The PPP models should be selected carefully; otherwise they may make the highway system costly and may cause optimal pavement design and selection of optimal rehabilitation strategies and timing of projects impossible [26, 27]. Early PMS did not have PPP models rather they evaluated only the current pavement conditions. Later, simplified PPP models, usually based on the engineering opinions on the expected design life of different M\&R actions, were introduced by considering age of the pavement as the only predictive variable [28]. The PPP modeling is explicitly complicated as it is very difficult to estimate incorporating a large number of parameters of pavement performance. A variety of approaches can be used to predict pavement performance such as regression, survivor curves, latent variable models, mechanistic models and Markov chain probabilistic models.

Probabilistic models recently have received considerable attention from pavement engineers and researchers. Typically, a probabilistic model is represented by the Markov transition process, which is a knowledge-based expert decision model for the prediction of pavement deterioration [25]. Knowing the 'before' condition' or state of pavement in probabilistic form, one can employ the Markov process to predict the 'after' state, again in probabilistic forms, for as many time steps as are desired [24]. The major challenge, facing the existing stochastic pavement performance models, includes difficulties in establishing transition probability matrices (TPMs).

TPMs are estimated using a non-linear programming approach. The objective function of TPMs is to maximize the absolute distance between the actual PCR versus age data points and the predicted PCR for the corresponding age generated by the Markov chain [23]. The 
assumption is that the pavement condition does not drop by more than one state in a single year. Thus, the pavement either stays in its current state or transits to the next lowest state in one year. The probability transition matrix has a diagonal structure. The pavement condition cannot transit from this state unless repair action is performed [23].

Several studies [24, 29] applies empirical-mechanistic pavement performance model to calculate PCR. George, et al. [24] executes various regression analyses to develop empiricalmechanistic performance model for the highways in Mississippi based on the pavement condition data during the period of 1986-1988. Several different models were constructed and evaluated. The evaluation was based on rational formulation and behavior of the model and on its statistical parameters. Exponential and power functions of both concave and convex shapes are identified as statistically significant.

The prediction models recognize the yearly equivalent single axle load (ESAL) and structural number (SN) are of only minor importance, while age being the most important factor of pavement performance modeling. George, et al. [24] argue that ESAL is the weakest link in the cumulative traffic computation because several questionable and dynamic input parameters (e.g. traffic count, the growth factor, the truck factor) are associated with the ESAL estimation. Traffic volume and truck factor is associated with the economic growth, land use changes, travel behavior, environmental condition and so on. George, et al. [24] apply the same argument for the exclusion of the environmental loads which include thermal effects, subgrade movements in expansive clays if applicable, freeze-thaw effects, and bitumen aging. George, et al. [24] find out that computational accuracy along with the direct influence of SN and asphalt concrete thickness on the mechanistic parameters (e.g. stress, strain and deflection) is the reason for its significance in the performance model.

Smadi and Maze [29] determine the PCR for the Iowa Interstate 80 based on the 10 years traffic data. The performance curve of PCR is assumed to be a function of only the total number of 18 kip equivalent single-axle loads (ESALs) that the pavement has experienced.

Traffic volumes, which are transferred into ESALs, can be calculated for each road link by applying four-step transportation modeling - trip production, trip distribution, modal split and choice analysis, and traffic assignment. Trip production is performed by relating the number or frequency of trips to the characteristics of the individuals, of the zone, and of the transportation network. Discrete choice models use disaggregate household or individual level data (personal, household, zonal and transportation network characteristics) to estimate the probability with which any household or individual will make trips. The discrete model is a stochastic model in which the probability of generating the trip is a function of a set of explanatory variables, such as: gender, age, personal and household income, occupation, family size, auto ownership, number and age of the children in the household, land use, residential density, accessibility and level of service of the roads. The outcome can then be aggregated to predict the number of trips produced.

Trip distribution models (e.g. growth factor models and gravity models) are used to predict spatial pattern of trips or other flows between origins and destinations. Modal choice model estimation and application are done to predict the zonal shares of trips by mode.

The Multinomial Logit (MNL) model relates the probability that a decision unit chooses a given alternative from a set of alternatives to the utility of these alternatives. The model choice model estimation and application can be done at either disaggregate or aggregate zonal level. The aggregate application predicts the zonal shares of trips by model. The disaggregate application is based on individual level data obtained from surveys or population synthesis. The 
explanatory variables of the modal utility are socio-economic characteristics of the travelers and the service attributes of the alternative modes.

The traffic assignment models (All-or-Nothing, STOCH, Incremental, Capacity Restraint, User Equilibrium, Stochastic User Equilibrium, and System Optimum) predict the network flows that are associated with future planning scenarios, and generates estimates of the link travel times and related attributes that are basis for benefits estimation and air quality impacts. The traffic assignment model is also used to generate the estimates of network performance that are used in the mode choice and trip distribution stages of many models.

\section{DYNAMIC PROGRAMMING PROCESS OF PAVEMENT MANAGEMENT SYSTEM}

The dynamic programming process starts with the calculation of the routine maintenance cost for each state condition in every family (categorized based on the pavement characteristics e.g. type, structure, construction history, condition, use, and rank) in a particular year. Routine maintenance is not feasible if $R_{i j k}=0$ or state condition $S_{j} \geq i$ for family $j . R_{i j k}$ is the feasibility indicator for alternative maintenance operation $k$ when in state $i$ of family $j . R_{i j k}=1$ if maintenance alternative is feasible and 0 for infeasible alternative. $S_{j}$ is the minimum allowable state for each $j$ family, i.e. the lowest state that the network manager will allow a particular family to deteriorate to before performing some major maintenance. The state vector of any period $n, S_{j n}$, is obtained by multiplying the initial state vector, $S_{0}$ by the transition matrix $\left(P_{i j}\right)$ raised to the power of $n$ [23].

For all feasible states, the cost of routine maintenance is obtained from $C_{i j k, N}=C_{i j k}$, where $k$ is the maintenance alternatives [23]. The cost of all feasible maintenance alternatives for year $N-n$ is given by Equation 1 [23].

$$
C_{i j k, N-n}^{*}=C_{i j k}+\left[P_{i j}^{1} * C_{i j^{1}, N-n+1}^{*}+\left(1-P_{i j^{1} 1}\right) * C_{2 j^{1}{ }^{1} N-n+1}^{*}\right] * \frac{1}{\left(1+i^{*}\right)}
$$

Where $P_{i j}$ is the Markov Transition probabilities for state $i$ (1..10 states) of matrix $j(1 \ldots$ $m$ families). $P_{i j}$ transition matrix probabilities are estimated using a non-linear programming approach which has its objective function the minimization of absolute distance between the actual pavement condition versus pavement age and the expected pavement condition. $C_{i j k}$ is the cost of applying treatment $k$ (1...n maintenance alternatives) to family $j$ in state $i$ [23].

The first part $\left(C_{i j k}\right)$, of the right side of Equation 1, is the immediate cost of routine

maintenance in year $n$. The second part $\left(\left[P_{i j}^{1} * C_{i j^{1}, N-n+1}^{*}+\left(1-P_{i j^{1} 1}\right) * C_{2 j^{1}, N-n+1}^{*}\right] * \frac{1}{\left(1+i^{*}\right)}\right)$ is the total expected cost to be incurred in the remaining years as a consequence of applying routine maintenance operations. This expected cost is obtained by identifying the probability of remaining in a given state and multiplying this probability by the expected cost of that state and then finding the associated probability of dropping a state if routine maintenance is applied and multiplying this by the expected cost of the lower state. This sum is then discounted by the effective interest rate, $i^{*}$, to calculate the present net value in the year $N-n$ [23].

The optimum maintenance strategy is then given by Equation 2 [23].

$$
C_{i j k, N-n}^{8}=\operatorname{Min}\left[C_{i j 1, N-n}, C_{i j k, N-n}\right] \text { for all } k \text {. }
$$


With the related optimal maintenance alternative to be performed for this $(i, j)$ family or state combination in year $N-n$ being the choice of $k$ that minimizes the cost in Equation 1. This backward recursion is performed for every successive year of the analysis period until the analysis for year 0 , or stage $N$, is reached. [23].

\subsection{Road user costs of pavement management system}

The objective function of life-cycle cost optimization is not only to reduce the maintenance costs but also to reduce the user costs. The road user costs are defined as the consequences of the periodic M\&R strategies of pavement section on the road users. Moreover, the vehicle, accident and time costs are directly associated with pavement condition deterioration. Vehicles costs for fuel, lubricants, tires, repairs and depreciation are proportionately related to the pavement surface condition. For example, fuel consumption is a function of vehicle's speed and the road geometry (mainly vertical upgrade and downgrade) [30]. Although an increase of international roughness index (IRI) has very insignificant impact on fuel consumption and lubricant costs, the impact of rutting is high during the wet season. In rain, when the ruts are full of water, the rolling resistance increases as a function of water depth. The average water depth is a result of cross-fall, rut depth, precipitation levels and intensity, traffic volume, and the driver's behavior. The increase in rolling resistance gives an increase in fuel consumption when driving on wet surfaces [30]. However, repair costs of vehicles are related to IRI rather than rutting. For example, the roughness can affect $50 \%$ of repair costs for passenger cars and $25 \%$ for heavy goods vehicles in Norway road network [30].

The cost of travel time is a product of value of time and the time to travel a certain distance. Traveling time is a result of traveling speed, which is the function of speed limit, road width, and curvature. Speed limit, road width, and curvature generate a speed of $70 \mathrm{~km} / \mathrm{hr}$ at an average roughness of 2.7 (IRI) [30]. The commuters also drive additional travel distances in order to avoid the maintenance links of the road network resulting in additional cost of travel time. The cost of travel time can be estimated by calculating the salary of the equivalent time spends on working hours.

The number of accidents within a road section is a product of various casual factors. The Transportation Association of Canada has categorized causal factors into three main groups: road geometry elements, environmental factors and human characteristics [31]. De Leur and Sayed [32] categorized the causal factors into three generic groups such as exposure, consequences and probability. Exposure has been defined as the extent of exposure to road accidents by road users [31]. Consequences can be expressed by the severity of road accidents and the probability was explained as the likelihood of accidents occurrence [31]. Sayyadi et al. [32] identify several road geometrics, traffic and environmental characteristics as the causal factors of road accidents. These are: individual car accident percentage, vehicle's speed, intersections per kilometer, severity of horizontal and vertical alignment, length of the road section, Annual Average Daily Traffic (AADT), light condition, weather-surface condition, road cross-section characteristics, and shoulder and land width.

The objective function (Equation 3) is also to minimize user cost along agency cost (maintenance cost plus initial or construction cost) with under budget constraints (Equation 4) [10].

$$
\operatorname{Min} \sum_{i} \Sigma_{j} \Sigma_{k} w_{i k}\left(C_{i j k, N-n}^{*}+U_{i k}\right) \forall \sum_{i} \Sigma_{k} w_{i k}=1
$$




$$
\begin{aligned}
& \sum_{k} w_{i k} \leq \varepsilon_{i}(1+\emptyset) \text { for all unacceptable } i \text { and } \sum_{k} w_{i k} \geq \varepsilon_{i}(1+\emptyset) \text { for all acceptable } i \text {. } \\
& B(1-\Omega) \leq \sum_{i} \sum_{j} \sum_{k} w_{i k}\left(C_{i j k, N-n}^{*}\right)-\beta \leq B(1+\Omega)
\end{aligned}
$$

Where $w_{i k}$ is the fraction of area of pavement in state $i$ with action $k$ applied, $B$ is the budget constraint per year, $\beta$ is the parametric analysis adjustment on budget constraint, $\Omega$ is the tolerance on budget constraint, $\varepsilon_{i}$ is the condition constraint for state $i$, $\emptyset$ is the tolerance on condition constraints [10].

\subsection{Drawbacks of Markov decision process}

The main drawback of Markov decision Process (MDP) approach is that it does not accommodate budget constraints [33]. Another important drawback of this approach is that pavement sections have to be grouped into a large number of roughly homogeneous families based on pavement characteristics [34]. A large number of families mean fewer sample of pavement sections in each family, which compromises the reliability and validity of the transition probability matrix (TPM) generated for each family [34]. There are equally large numbers of $M \& R$ treatments for each family of pavement sections. It is suggested that all pavement sections should be categorized into small numbers of families. As the MDP addresses the performance evaluation of the pavement section as a group, it is not possible to address the performance condition of individual pavement section. Similarly, the optimization programming of M\&R strategies are determined for a group of pavement sections rather than an individual section under a given budget. Moreover, the optimization programming of M\&R strategies are calculated from the steady-state probabilities. However, in reality, the pavements under a given maintenance policy usually takes many years to reach the steady state and the proportion of the pavements are changing year by year. Therefore, the use of steady-state probabilities in the optimization objective function does not fully reflect reality, especially when this transition period is very long [34].

\subsection{Drawbacks of project-based pavement management system and existing practices to deal with these problems}

The project-based modeling approach is based on the analysis of historical performance data stored in the database to develop model coefficients for individual pavement sections. For each individual section, the available historical performance data since the last rehabilitation or construction is analyzed to determine the model that matches the observed performance of the section and thus predict the future performance. The cost effectiveness, later on, is derived from the prediction performance curve. The pioneer of project-based optimization method for pavement M\&R strategies and projects is Arizona DOT [34].

Thompson et al. [10] divide the Finnish highway network into six regional class subnetworks and propose individual optimization model for each of the sub-network group rather than for an individual road segment. Each model is classified among four dimensions - bearing capacity, pavement defects, rutting, and pavement roughness.

The proposed models by Arizon DOT and Thompson et al. [10] solve the optimization problem at an individual or group of pavement section, however, the deterioration uncertainty 
has not yet been resolved. The required budget should treat the uncertainty of deterioration carefully by incorporating dynamic characteristics of road data. The traffic loads on the pavement are uncertain and dynamic in character and need to be included in the maintenance budget.

Butt et al. [35] introduce different duty cycles to allow the changes in traffic loads and maintenance polices over the pavement life. This nonhomogeneous Markov model divides the life of the pavement into different zones assuming a constant rate of deterioration for each of these zones. A homogeneous Markov chain and a separate TPM are developed for each zone. On the other hand, a nonhomogeneous Markov chain has been used for transition from one zone to another [35].

A research project titled 'Maintenance Cost Prediction for Road' under the cooperative Research Centre (CRC) for Construction Innovation has also developed a method that takes into account the variability and uncertainties of road data in investment analysis [36]. Piyatrapoomi et al. [36] identify the variability parameters of the predicted budget, which includes rut depth, AADT, initial roughness and pavement strength [36]. The variability of these parameters was quantified by probability distributions, means and standard deviation for each category. Piyatrapoomi et al. [36] identify the best fitted probability distribution functions (pdf) for IRI, rut depth, and AADT of the Queensland road network are Beta General, log normal and exponential distributions, respectively. Piyatrapoomi et al. [36] apply Latin-hypercube sampling technique to simulate the variability of above-mentioned parameters.

Another major drawback to practice project-based optimization is that the complexity of pavement management problems increases exponentially with the size of the problem. For example, the number of possible solutions for project-based optimization problems is $M^{T}$, where $M$ is the number of maintenance actions to be considered and $T$ is the number of years (or periods) in the analysis period. If the solution space size for a single section given a certain analysis period and number of available maintenance actions (project level) is $C$, the solution space size of the network problem, assuming $S$ similar sections, is $\boldsymbol{C S}$. With the general projectlevel complexity, therefore, this equates to $\left(A^{T}\right)^{C S}[37]$.

Pilson et al. [37] propose a genetic algorithm (GA) optimization model to overcome this complexity. The GA optimization model considers each analysis period as a 'gene' and maintenance actions as 'allele' values. A chromosome represents a maintenance strategy for a particular period. The second step of GA optimization model is to measure the 'fitness' of the chromosomes [37]. However, the major drawback of GA optimization model for PMS is that the chromosome has one gene for each pavement section and each gene can take on any value from that section's efficient set. This decomposition of the network problem relies on the independence of the project-level problems, which is not theoretically acceptable for annual budgets [37].

The project-based PMS is still struggling with the large size of problems and solutions. Although we can solve this problem by GA optimization model, there is a decomposition problem of the independence of the project-based PMS. This is not suitable under an annual budget for the whole road network. Moreover, the above-mentioned models optimize the PMS by minimizing the cost, ignoring the maximization of pavement condition. 


\subsection{Multi-criteria pavement management system}

The construction of new road and maintenance of exiting road network have significant impacts on the surrounding locations such as changes in economic activities, and social and cultural changes. PMS should include the effect of M\&R strategies on other road users and surrounding locations such as residents in close proximity from the road, industrial settlements, trade centers, etc. [38]. The Action de Préparation, d'Accompagnement, et de Suivi (APAS) transport research project, funded by the European Commission, develops some indicators for the choice of transport projects using decision criteria optimization [39]. The indicators are developed through simplified multi-criteria analysis (MCA) techniques such as Elimination et Choix Traduisant la Realité (ELECTRE III) [40] and the analytical hierarchy process (AHP) [51]. Highway Development and Management Tool (HDM-4) applies AHP method to integrate multi-criteria factors with the PMS [39].

Cafiso et al. [39] identify ride comfort and environmental factors as the criteria of PMS along with agency costs and user costs. The ride comfort is defined as ride number $(R N)$ values by National Cooperative Highway Research Program (NCHRP). Ride number $(R N)$ values for alternative $k$ of the section $j$ are obtained from $I R I(\mathrm{~m} / \mathrm{km})$. The environment parameter for a road segment can be calculated by deriving air quality index (AQI). AQI is a function of emission value of the air pollution substance for an alternative of each section at a particular year, average annual daily traffic for each section at a year, length of the section, concentration limits of a single air pollution substance [39].

Socio-economic development parameter can have significant relation with the PMS. The residents, in close proximity to the invested road infrastructure, may achieve significant socioeconomic benefits from the PMS strategies. The characteristics of development parameters of urban area can be demographic characteristics, economic characteristics, social and community characteristics, transportation facilities, urban services and facilities, and environmental characteristics.

Instead of project-based PMS, this paper proposes a PMS for different categories of road groups with different pavement performance curves for each group.

The objective functions of multi-criteria PMS are given by Equation 12 and 13.

$\operatorname{Min} \sum_{i} \sum_{j} \sum_{k} w_{i k}\left(C_{i j k, N-n}^{*}+U_{i k}\right) \forall \sum_{i} \sum_{k} w_{i k}=1$

$\sum_{k} w_{i k} \leq \varepsilon_{i}(1+\emptyset)$ for all unacceptable $i$ and $\sum_{k} w_{i k} \geq \varepsilon_{i}(1+\emptyset)$ for all acceptable $i$.

Subject to : $\sum_{n=1}^{N} \sum_{i=1}^{I} L_{i} Q_{n, i} \geq(L O S) \sum_{i=1}^{I} L_{i}$

$\operatorname{Max} \sum_{n=1}^{N} \sum_{i=1}^{I}\left(W_{i} * L_{i} Q_{n, i}+W_{2} * M C I_{i}\right)$

$M C I_{i}=f$ (comfort, environment, socio - economic development criteria)

Subject to: $B(1-\Omega) \leq \sum_{i} \sum_{j} \sum_{k} w_{i k}\left(C_{i j k, N-n}^{*}\right)-\beta \leq B(1+\Omega)$

Where $Q_{n, i}=$ Condition Index for asset $i$ on year $n ; M C I_{i}=$ multi-criteria index for asset $i$, and $W_{1}$ and $W_{2}$ are the weights of the condition index and multi-criteria index. 


\section{CONCLUSION}

The pavement management systems incorporate the economic assessment of trade-offs between competing maintenance and rehabilitation alternatives. The conventional techniques are capable of finding the optimal path to take full advantage of cost-effectiveness of individual treatments, however, incapable of addressing safety, condition, congestion, pollution and social cost. The objective of this paper is to outline a conceptual framework of a pavement management system (PMS) for the road network of Montreal City.

This paper initially describes the life-cycle cost analysis as the economic optimization model for PMS. The methods of appropriate and effective pavement performance modeling are discussed as the pavement deterioration modeling is the foundation for the long-term analysis of PMS. The framework of integrated land use and transportation (ILUT) modeling, applying fourstep transportation modeling, is explained to predict the future traffic volume during the lifecycle of pavement. ILUT modeling is discussed because the predicted traffic volumes of each segment of road network are transferred to Equivalent Single Axle loads (ESALs).

The proposed dynamic programming process of PMS points out the drawbacks of the Markov decision process of network-based PMS and is brokering the project-based PMS. The project-based PMS is still struggling with exponentially increasing size of problems and M\&R solutions. This study proposes a PMS for different categories of road groups with different pavement performance curves for each group. This paper also proposes the incorporation of road user costs and socio-economic development of the residents (in close proximity to road infrastructure) along with agent costs in the PMS. This dynamic PMS will manage continuous aggregate behavior of transportation system and can solve optimization problems of pavement management at any time interval.

\section{References}

1. Hudson, W. R., R. Haas, and W. Uddin. Infrastructure Management. New York: McGraw Hill, 1997.

2. Haas, R., and W. R. Hudson. Pavement management systems. New York, N.Y.: McGraw-Hill, 1978.

3. Watanatada, T., Harral C.G., Paterson W.D.O., Dhareshwar A. .M., Bhandari A., and K. Tsunokawa. The Highway Design and Maintenance Standard Model. Washington D.C.: The John Hopkins University Press, 1987.

4. NCHRP. Analytical tools for Asset Management. Report 545, Washington D.C.: Transportation Research Board, National Cooperative Research Program, 2005.

5. Robelin, C. A., and S. M. Madanat. "History-dependent bridge deck maintenance and replacement optimization with Markov decision processes." Journal of Infrastructure Systems 13, no. 3 (2007): 195-201.

6. Chootinan, P., A. Chen, M. R. Horrocks, and D. Bolling. "A multi-year pavement maintenance program using a stochastic simulation-based genetic algorithm approach." Transportation Research Part A 40, no. 9 (2006): 725-743 .

7. NAMS. International Infrastructure Management Manual. New Zealand: National Asset Management Steering Group, 2006.

8. Donaghy, K. P., and L. A. Schintler. A Prototype Dynamic Transportation Network Management Model. Final report, Delaware : Delaware Department of Transportation, 1993. 

2012.

9. City of Montreal, C. 2012 Operating Budget at a Glance. Montreal: City of Montreal,

10. Thompson, P. D., L. A. Neumann, M. Niettinen, and A. Talvitie. "A Micro-Computer Markov Dynamic Programming System for Pavement Management in Finland." Pavement Management. 1987. http://www.pavementmanagement.org/ICMPfiles/1987042.pdf (accessed June 28, 2013).

11. Ockwell, Anthony. Pavement Management: Development of a Life Cycle Costing Technique. Occasional Paper 100, Bureau of Transport and Communications Economics, Department of Transport and Communications, Canberra: Australian Government Publishing Service, 1990.

12. Potter, D. W., and W. R. Hudson. "Optimisation of Highway Maintenance Using Highway Design Model." Australian Road Research 11, no. 1 (1981).

13. Karlsson, Charlie, Borje Johansson, William P. Anderson, and Kiyoshi Kobayashi. "Infrastructure Meansurement and Management: An Introduction." In The Management and Measurement of Infrastructure: Performance, Efficiency and Innovation, edited by Charlie Karlsson, William P. Anderson, Borje Johansson and Kiyoshi Kobayashi, 1-24. Massachusetts, USA: Edward Elgar Publishing Inc., 2007.

14. Christensen, P.N., G.A. Sparks, and K.J. Kostuk. "A Method-based Survey of Life Cycle Costing Literature Pertinent to Infrastructure Design and Renewal." Canadian Journal of Civil Engineering 32 (2005): 250 - 259.

15. Uddin, W., R. F. Carmichael, and W. R. Hudson. Life Cycle Analysis of Pavement Management Decision Making. Final Report, Washington D.C.: Department of Transportation, 1986.Voogd, H. Multicriteria Evaluation for Urban and Regional Planning. London: Pion Limited, 1983.

16. Carnahan, J. V. "Analytical Framework for Optimizing Pavement Maintenance." Journal of Transportation Engineering 114, no. 3 (1988): 307-322.

17. Kher, R., and W. Cook. "PARS-the MTC Model for Program and Financial Planning in Pavement Rehabilitation." North American Pavement Management Conference. Washington D.C.: Ontario Ministry of Transportation and Communication, U.S. Federal Highway Administration, 1985. 6.24-6.40.

18. Scullion, T., and A. Stein. "Predicting Maintenance and Rehabilitation Needs for the State of Texas." North American Pavement Management Conference. Washington D.C.: Ontario Ministry of Transportation and Communication, U.S. Federal Highway Administration, 1985. 5.54-5.64.

19. Artman, D., and J. Liebman. "Optimization of Long-range Major Rehabilitation of Airfield Pavements." Transportation Research Record 938, no. January (1983): 1-11.

20. Winston, W. Operations Research: Applications and Algorithms. 4th. Belmont, California: homson-Brooks/Cole, 2004.

21. Golabi, K., Kulkarni.R., and G. Way. "A statewide pavement management system." Interfaces 12, no. 6 (1982): 5-21.

22. Carnahan, J., W. Davis, M. Shahin, P. Keane, and M. Wu. "Optimal Maintenance Decisions for Pavement Management." Journal of Transportation Engineering 113, no. 5 (1987): 554-572.

23. Feighan, K. J., M. Y. Shahin, and K. C. Sinha. "Dynamic Programming Approach to Optimization for Pavement Management Systems." Transportation Research Board (TRB) 
Committee AFD10 on Pavement Management Systems. 1987. http://www.pavementmanagement.org/ICMPfiles/1987039.pdf (accessed June 28, 2013).

24. George, K. P., A. S. Rajagopal, and L. K. Lim. "Models for Predicting Pavement Deterioration." Transportation Research Record: Journal of the Transportation Research Board (Transportation Research Board of the National Academies) 1215 (1989): 1-7.

25. Li, N., R. Haas, and W-C. Xie. "Investigation of Relationship Between Deterministic and Probabilistic Prediction Models in Pavement Management." Transportation Research Record 1592 (1997): 70-79.

26. Johnson, K.D., and K.A. Cation. "Performance Prediction Development Using Three Indexes for North Dakota Pavement Management System." Transportation Research Record (Transportation Research Board, National Research Council, Washington, D.C.) 1344 (1992): 22-30.

27. Attoh-Okine, N. O. "Analysis of Learning Rate and Momentum Term in Backpropagation Neural network Algorithm Trained to Predict Pavement Performance." Advances in Engineering Software 30 (1999): 291-302.

28. Kulkarni, R. B., and R. W. Miller. "Pavement Management Systems Past, Present, and Future." Transportation Research Record 1853 (2002): 65-71.

29. Smadi, O. G., and T. H. Maze. "Network Pavement Management System Using Dynamic Programming: Application to Iowa State Interstate Network." . San , Nation." Third International Conference on Managing Pavements. Antonio, Texas: Transportation Research Board, National Research Council, 1994.

30. Haugodegard, T., J. M. Johansen, D. Bertelsen, and K. Gabestad. "Norwegian Public Roads Administration: A Complete Pavement Management System in Operation." 3rd International Conference on Managing Pavements. San Antonio, Texas: National Research Council, Transportation Research Board, 1994.

31. Sayyadi, G., L.E. Amador, and M.S.R. Amin. "A Multivariate Analysis of Road Severity Accident Index." 23rd Canadian Multidisciplinary Road Safety Conference. Montréal, Québec: The Canadian Association of Road Safety Professionals, May 26-29 2013.

32. De Leur, P., and T. Sayed. "Development of a Road Safety Risk Index." Transportation Research Record: Journal of the Transportation Research Board (Transportation Research Board of the National Academies) 1784 (2002): 33-42.

33. Liebman, J. "Optimization Tools for Pavement Management." North American Pavement Management Conference. Washington D.C.: Ontario Ministry of Transportation and Communication, U.S. Federal Highway Administration, 1985. 6.6-6.15.

34. Li, Y., A. Cheetham, S. Zaghloul, K. Helali, and W. Bekheet. "Enhancement of Arizona Pavement Management System for Construction and Maintenance Activities." Transportation Research Record: Journal of the Transportation Research Board 1974 (2006): 26-36.

35. Butt, A. A., M. Y. Shahin, S. H. Carpenter, and J. V. Carnahan. "Application of Markov Process to Pavement Management Systems at Network Level." 3rd International Conference on Managing Pavements. San Antonio, Texas: Transportation Research Board, National Research Council, 1994. 159-172.

36. Piyatrapoomi, N., A. Kumar, N. Robertson, and J. Weligamage. "A Framework for Risk-based Analysis of Investment in Maintenance and Rehabilitation of Large Road Networks." Joint International Conference on Computing and Decision Making in Civil and Building Engineering, June 14 - 16. Montreal, 2006. 
37. Pilson, C., R. Hudson, and V. Anderson. "Multiobjective Optimization in Pavement Management by Using Genetic Algorithms and Efficient Surfaces." Transportation Research Record 1655 (1999): 42-48.

38. Cafiso, S., A. Di Graziano, H. R. Kerali, and J. B. Odoki. "Multicriteria Analysis Method for Pavement Maintenance Management." Transportation Research Record 1816 (2002): 73-84.

39. European Commission, E. Evaluation Methods: APAS Road Transport Research Project. Luxembourg: European Commission, 1996.

40. Hokkanen, J., and P. Salminen. "ELECTRE III and IV Decision Aids in an Environmental Problem." Journal of Multi-Criteria Decision Analysis 6, no. 4 (1997): 215-226. 